\title{
Kirche und Kommunikation
}

Zur bleibenden Aktualität von COMMUNIO ET PROGRESSIO

Sonderheft zum

Welttag der Sozialen

Kommunikationsmittel 1991

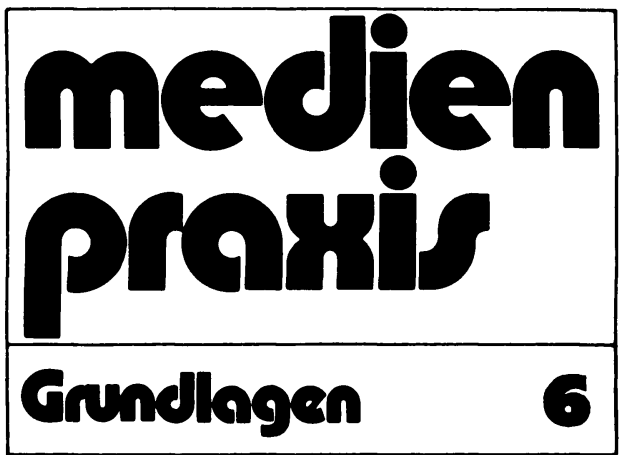

Inhalt

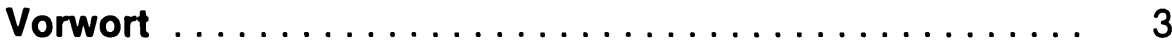

Bernhard Laux:

Kommunikation und Öffentlichkeit

Verständnis - Anliegen - Konsequenzen .......... 5

Hans Gasper:

Die Kernaussagen von Communio et Progressio

Theologische und sozialethische Überlegungen . . . . . . 24 



\section{Kommunikation und Öffentlichkeit}

Verständnis - Anliegen - Konsequenzen

1. Ein Dokument im Geist und Auftrag des II. Vatikanischen Konzils .......................... 5

2. Das Verständnis von Kommunikation und Öffentlichkeit . . . . . 7

2.1 Kommunikation und Öffentlichkeit - Verständigung statt Zwang und Manipulation ................ 7

2.2 Die Möglichkeit des Mißbrauchs von Massenkommunikation und die Notwendigkeit sozialethischer Reflexion . . . . 9

3. Anforderungen an die Massenkommunikation ............ 10

3.1 Grundanforderung: Sicherung des Forumcharakters ..... . . 11

3.2 Konsequenzen für die Medienstrukturen: Institutionsethik . . 11

3.3 Anforderungen an die einzelnen: Handlungsethik . . . . . . . 13

3.4 Medienpädagogik als Hilfe für sachgerechtes und damit

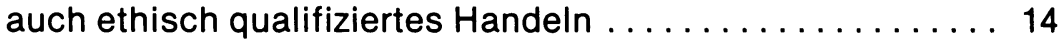

4. Die Rolle der Kommunikationsmittel im Leben der Kirche . . . . . . 15

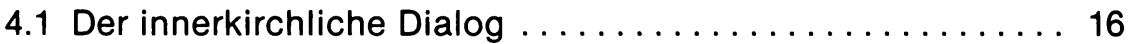

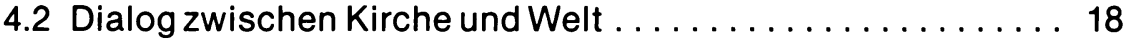

5. Würdigung und Ausblick ................... 20

\section{Ein Dokument im Geist und Auftrag des II. Vatikanischen Konzils}

Das II. Vatikanische Konzil praktizierte eine für die Kirche neue Form von Kommunikation und Öffentlichkeit. Der kommunikative und dialogische Umgang diente nicht der bloßen „Öffentlichkeitsarbeit“. Er ist in der Sache des Evangeliums selbst begründet und wird für die Erneuerung der Kirche nach innen und nach außen als wesentlich erachtet.

So ist es durchaus konsequent, daß es als erstes Konzil auch ein Dekret über die Massenkommunikation „Inter mirifica“ verfaßt hat, das, zusammen mit der Liturgiekonstitution, als erstes Konzilsdokument verkündet wurde. Dadurch konnte es an der Entwicklung des Konzils nicht voll teilhaben und blieb hinter dem im Verlauf des Konzils erreichten Stand zurück. ${ }^{1}$

Die Pastoralinstruktion „Communio et Progressio“ jedoch kann nun aus der ganzen Fülle der Theologie des II. Vaticanums schöpfen und es 
bestand Zeit und Gelegenheit, den Stand der Publizistikwissenschaft zur Kenntnis zu nehmen und theologisch zu reflektieren. Aus dieser Verbindung erwuchs ein beachtliches Dokument, das auch heute noch beachtenswert, herausfordernd und weiterführend ist.

Das II. Vaticanum überwindet in einem vertieften Verständnis der Frohbotschaft alle Versuche und Versuchungen, die Freiheit gesellschaftlicher Kommunikation von einer vorgängig gewußten Wahrheit her zu beschränken - was nur in totalitären und antichristlichen Strukturen enden kann. So wie in "Gaudium et spes" die plurale und demokratische Gesellschaft mit ihren Freiheitsrechten, in "Dignitatis humanae“ explizit die Religionsfreiheit, so werden in "Communio et Progressio“ Kommunikations- und Medienfreiheit, die die Kirche nun wahrlich aufs schärfste verurteilt hatte, nicht nur akzeptiert, sondern als Fortschritt verstanden, der von Bedeutung für das Wachsen des Reiches Gottes ist. Wo Massenmedien zu „communio et progressio“, also zu „Einheit und Fortschritt" der menschlichen Gesellschaft beitragen, fördern sie etwas nicht nur „Weltliches“. Das Konzil überwindet gerade dieses Bereichsdenken von Welt und Glaube, von Glaube und Politik. Wo Massenmedien zu Gemeinschaft und Fortschritt der menschlichen Gesellschaft beitragen, da bringen sie Berufung und Würde der menschlichen Person zur Geltung, arbeiten sie mit beim Voranschreiten des Reiches Gottes und sind im Prozeß der Evangelisierung begriffen.

„Gemeinschaft und Fortschritt der menschlichen Gesellschaft sind die obersten Ziele sozialer Kommunikation und ihrer Instrumente, wie der Presse, des Films, des Hörfunks und des Fernsehens“ (CeP 1).

„Besseres Verständnis und Rücksichtnahme unter den Menschen, Hilfsbereitschaft und schöpferische Zusammenarbeit, wie sie durch die soziale Kommunikation in erstaunlichem Maße gefördert werden können, sind in der Tat Ziele, die mit denen des Gottesvolkes nicht nur im Einklang stehen, sondern von daher sogar noch tiefer gesichert und vervollkommnet werden. „Denn die Förderung der Einheit hängt ja mit der innersten Sendung der Kirche zusammen, da diese ,in Christus gleichsam das Sakrament, d.h. Zeichen und Werkzeug für die innigste Vereinigung mit Gott wie für die Einheit der ganzen Menschheit ist “ “ (CeP 18). 


\section{Das Verständnis von Kommunikation und Öffentlichkeit}

„Communio et Progressio“ gelingt es als erstem kirchlichen Dokument, ein Verständnis der Massenkommunikation von ihrer gesamtgesellschaftlichen Bedeutung her zu entwickeln: Massenmedien bilden ein Forum, auf dem das Gespräch und die Auseinandersetzung der Gesellschaft über den Zustand und die notwendige Entwicklung des Gemeinwesens stattfinden kann. Damit ist ein Ansatzpunkt für die Ethik der Massenkommunikation gewonnen: Massenkommunikation insgesamt und die Handlungen der daran in verschiedener Weise beteiligten Menschen müssen sich am Anspruch messen lassen, ob sie der Erfüllung dieser gesellschaftlichen Aufgabe dienen. Einzelne, dem katholischen Denken anstöBige Medieninhalte, können - wenn man Massenkommunikation in ihrer gesellschaftlichen Dimension begriffen hat nicht mehr Schwerpunkt der Auseinandersetzung sein.

\subsection{Kommunikation und Öffentlichkeit - Verständigung statt Zwang und Manipulation}

Menschliches Zusammenleben in Gesellschaft erfordert, daB die Handlungen der einzelnen Gesellschaftsmitglieder aufeinander abgestimmt werden. Diese Handlungskoordination kann - stellen wir uns der Einfachheit halber zwei Handlungspartner vor - auf Einverständnis beruhen, das auf der Grundlage von gemeinsamen Überzeugungen bereits besteht oder in Kommunikation zwanglos hergestellt werden kann. Wir haben es hier mit einem wechselseitigen Prozeß zu tun. Zwei Interaktionspartner bauen sich jeweils gegenseitig und einvernehmlich in ihre Handlungspläne ein und wissen wechselseitig davon. Handlungskoordination kann aber auch durch Einflußnahme auf den Interaktionspartner erreicht werden. Er soll sich so verhalten, wie es meinen Plänen entspricht und dazu wirke ich in geeigneter - nicht rein kommunikativer - Weise auf inn ein: Beispielsweise durch Drohungen oder Versprechungen oder durch eine Steuerung von Kommunikation, die den anderen desorientiert: je nachdem wie es zur Durchsetzung der eigenen Interessen günstig ist, werden dem anderen Informationen gezielt gegeben oder vorenthalten, Sprache wird beim Versuch der EinfluBnahme meistens beteiligt sein. (Selbst ein Bankraub kommt ohne einen Rest sprachlicher Interaktion nicht aus: „Geld her") Aber sie ist defizient gegenüber ihrer Vollgestalt, da Kommunikationsvoraussetzungen wie vor allem Zwanglosigkeit und Wahrhaftigkeit verletzt werden.

Kommunikation zielt ihrem innersten Wesen nach auf ein zwangloses, freies Einverständnis, auf Verständigung, obwohl wir natürlich wissen, 
daß die realen Kommunikationen dieses Ziel oft sehr weit verfehlen. „Über Kommunikation im gesellschaftlichen wie im privaten Bereich soll Konsens, Einheit und Gemeinschaft erreicht werden." 2 So ist Kommunikation ihrem grundlegenden Anspruch nach das Gegenstück von Zwang und Manipulation. Auch das verheißene Reich Gottes können wir uns wohl auch als reine, ungebrochene Kommunikation vorstellen. Abstimmung der Handlungen ist nicht nur auf der Ebene direkter personaler Interaktion, sondern auch auf der Ebene der Gesamtgesellschaft und ihrer Teilbereiche notwendig. Auch hier ist Kommunikation wesentliche Voraussetzung einer zwangfreien Koordination. Die Gestaltung des gesellschaftlichen Zusammenlebens ist Gegenstand dieser Kommunikation. Sie ist auf Massenmedien angewiesen, auch wenn nur ein Teil der gesellschaftlichen Kommunikation darüber vermittelt ist. Sie findet auch in Parlamentssitzungen, kirchlichen Stellungnahmen, Demonstrationen, Wahlen und vielen anderen Formen statt. Demokratie ist letztlich nichts anderes als eine genau geregelte Form der Kommunikation, in der Gesellschaftsmitglieder gemeinsame Angelegenheiten beraten und Entscheidungen treffen. Im demokratischen Staat regiert das Volk sich selbst, wenn auch vermittelt über repräsentative Instanzen. Deswegen sind Kommunikationskanäle zwischen den gesellschaftlichen Gruppen und zu den politischen Organen von großer Bedeutung.

„Die neue Technik für den Austausch unter den Menschen versammelt die Zeitgenossen sozusagen um einen runden Tisch. So kommen sie in dem Streben nach Brüderlichkeit und gemeinsamem Handeln miteinander ins Gespräch. Denn durch diese Instrumente wird das tägliche Gespräch der einzelnen aufgenommen, angeregt und weithin verbreitet. So wird das öffentliche Gespräch der ganzen Gesellschaft durch diese Medien ermöglicht und überall vernehmbar. Der so vermittelte Fluß der Nachrichten und Meldungen bewirkt in der Tat, daß alle Menschen auf dem ganzen Erdkreis wechselseitig Anteil nehmen an den Sorgen und Problemen, von denen die einzelnen und die ganze Menschheit betroffen sind. Das sind notwendige Voraussetzungen für das Verstehen und die Rücksichtnahme untereinander und letztlich für den Fortschritt aller“ (CeP 19).

Im Bild vom „runden Tisch“ drückt sich aus, was Massenmedien im besten Falle leisten können und warum diese Leistung für die Gesellschaft wichtig ist. (Nach den Umwälzungen in verschiedenen Staaten Osteuropas und in der DDR mit ihren „runden Tischen“ verstehen wir dieses Bildes besser und erkennen seinen Realitätsgehalt.) Es macht 
deutlich, daß wir Massenkommunikation nicht zureichend erfassen, wenn wir uns vorstellen, daß hier einer eine Mitteilung an viele richtet (z. B. eine Nachrichtenredaktion an viele Leser oder Zuhörer/Zuschauer). Dieser Prozeß ist nur ein Teil dessen, was Massenkommunikation ausmacht. Massenmedien ermöglichen eine gesellschaftliche Kommunikation über wichtige Fragen. Verschiedene Gruppen bringen ihre Positionen zu einem aktuellen Themenfeld in die gesellschaftliche Kommunikation ein, z. B. durch Reden, Presseerklärungen oder Demonstrationen; diese werden von den Massenmedien aufgegriffen, andere Gruppen antworten darauf, so da $B$ in den Massenmedien eine gesellschaftliche Diskussion stattfindet, die von allen Bürgern verfolgt werden kann und sie zu eigener Entscheidung und möglicherweise zu eigenem Engagement herausfordert. Die Massenmedien tragen die Erwartungen, Positionen und Argumente der Gesellschaft an die politischen Instanzen heran. Massenmedien stellen so ein Forum für das gesellschaftliche Gespräch zur Verfügung.

„Die Kommunikationsmittel bilden gewissermaßen ein öffentliches Forum, auf dem das Gespräch der Menschen hin und her geht. Die ÄuBerung und der Kampf der verschiedenen Meinungen in der Öffentlichkeit greift tief in das Leben der Gesellschaft ein, bereichert es und beschleunigt ihren Fortschritt" (CeP 24).

Aller Kommunikation - der gesellschaftlichen, über Medien vermittelten, wie der direkten, personalen - aber auch der Kommunikationsform ,Demokratie“ wohnt das Ziel einer offenen und freien Verständigung in Abgrenzung zu Zwang und Manipulation inne. (Wir sehen an negativen Beispielen, daB dort wo politischer Zwang herrscht, Kommunikation beschränkt werden muß und deformiert ist.) Kommunikation ist insofern niemals nur Mittel, sondern auch selber Ziel: Daß möglichst viel Kommunikation sei und möglichst wenig Zwang und Manipulation.

\subsection{Die Möglichkeit des Mißbrauchs von Massenkommunika- tion und die Notwendigkeit sozialethischer Reflexion}

Allerdings dürfen wir uns keinen Illusionen darüber hingeben, daß sowohl unsere direkte wie unsere gesellschaftliche Kommunikation von diesem Ziel weit entfernt sind. Auch da wo geredet wird, werden häufig Kommunikationsvoraussetzungen verletzt, so daB Kommunikation nicht der Verständigung und Anerkennung des anderen, sondern der Ausübung von Macht und der Manipulation dient. „Communio et 
Progressio" formuliert diesen Sachverhalt sehr deutlich und deutet inn theologisch:

„Die Kommunikationsmittel können zwar viel zur Einheit unter den Menschen beitragen. Unwissenheit und Mangel an gutem Willen lassen jedoch den Gebrauch dieser Mittel ins Gegenteil verkehren: die Menschen verstehen sich noch weniger, entzweien sich noch mehr, und die schlimmen Folgen werden ärger. Denn nur zu oft macht man die Erfahrung, daß durch eben diese Kommunikationsmittel die höchsten Werte des menschlichen Lebens verneint oder verfälscht werden. In diesen verhängnisvollen Erscheinungen sieht der Christ einen Hinweis darauf, daß der Mensch erlöst und befreit werden muß von der Sünde, die durch die Urschuld in die Menschheitsgeschichte eintrat“ (CeP 9).

Das Wissen um die Störung menschlicher Kommunikation, die erst im Reich Gottes von Grund auf und endgültig behoben sein wird, entlastet uns nicht vom Bemühen um eine Form von Massenkommunikation, die ihren Beitrag zur Humanisierung menschlichen Zusammenlebens leistet. Hieraus ergibt sich die Notwendigkeit zu sozialethischer Reflexion.

\section{Anforderungen an die Massenkommunikation}

Aus dem Grundverständnis der gesellschaftlichen Kommunikation sowie der Bedeutung der Medien hierfür entwickelt „Communio et Progressio“ Grundsätze für die Massenkommunikation. Da sie ein kompliziertes System aus technischen Einrichtungen, rechtlichen Regelungen, organisatorischen Strukturen, beruflichem Handeln von Kommunikatoren (z. B. Journalisten) und vielen anderen Berufsgruppen und dem Handeln der Rezipienten (Leser, Zuhörer, Zuschauer) ist, kann diese ethische Reflexion nicht in einigen wenigen Sollensätzen enden. "Communio et Progressio" formuliert einige grundlegende Anforderungen, die gesellschaftliche Kommunikation erfüllen muß, damit sie die dargestellten positiven Wirkungen erbringen kann. Daraus ergeben sich

- Anforderungen an die institutionelle Struktur der Massenkommunikation (Kommunikationsrechte und ihre politische Sicherung; Finanzierung; Organisations- und Kontrollstrukturen), die im wesentlichen (medien)politisches Handeln betreffen und damit zunächst die Politiker, im letzten aber in einer Demokratie alle Staatsbürger.

- Handlungsanforderungen an die an der Massenkommunikation Beteiligten, an Kommunikatoren und Rezipienten. 


\subsection{Grundanforderung - Sicherung des Forumcharakters}

Massenmedien können ein Forum schaffen, auf dem die gesellschaftliche Kommunikation stattfinden kann. Dieses Forum wirklich herzustellen, ist die ethische Grundforderung. Dazu ist Freiheit der Kommunikation notwendig, die Freiheit der Artikulation wie der Information umfaßt und Medienfreiheit selbstverständlich einschließt.

„Damit öffentliche Meinung sich nach den ihr eigenen Entstehungsgesetzen bilden kann, muß der Gesellschaft grundsätzlich der Zugang zu den Quellen und Kanälen der Information offenstehen und die Freiheit der Meinungsäußerung gewährt sein. Meinungsfreiheit sowie das Recht, zu informieren und informiert zu werden, bedingen einander notwendig. Johannes XXIII., Paul VI. und das II. Vatikanische Konzil haben das Recht auf Information klar und deutlich herausgestellt, da es für den Menschen und unsere moderne Gesellschaft wesentlich ist" (CeP 33).

"Sogenannte pluralistische Gesellschaften wissen sehr wohl, was die Möglichkeit der freien Verbreitung von Nachrichten und Meinungen für die aktive Teilnahme der Bürger am Leben der Gesellschaft wert ist. Darum schützen sie diese Freiheit durch Gesetze. Die Allgemeine Erklärung der Menschenrechte hat die Kommunikationsfreiheit als Grundforderung herausgestellt. Diese schlieBt notwendig die Freiheit der Instrumente der sozialen Kommunikation ein“ (CeP 46).

\subsection{Konsequenzen für die Medienstrukturen: Institutionsethik}

Die strukturellen Rahmenbedingungen sollen diesen Forumscharakter sichern, so daB Kommunikations- und Medienfreiheit als gleiche Freiheit allen gesellschaftlichen Teilgruppen zukommt. Ihre Teilnahme am Gespräch der Gesellschaft am „runden Tisch“ muß unabhängig von Wirtschaftskraft und politischer Macht gewährleistet sein.

„Im Einklang mit diesen Grundsätzen sind Freiheit der Kommunikation und Recht auf Information durch Gesetze zu schützen und ihr ungehinderter Gebrauch gegen Gewalt sowie gegen jeden wirtschaftlichen, politischen und ideologischen Druck ausreichend abzusichern. Den Staatsbürgern muß durch Gesetz eine volle Kontrollmöglichkeit der gesamten Verwaltung von Kommunikationsmitteln garantiert werden, besonders dann, wenn ein Monopol, und erst recht, wenn ein staatliches besteht. Es unterliegt heute keinem Zweifel, daB eine Kommunikationsgesetzgebung erforderlich ist. Sie muB eine ausreichende 
Vielfalt in den Medien wirksam schützen vor dem Druck wirtschaftlicher Marktgesetze, der eine übermäßige Konzentration zu erzwingen droht. Ebenso muß sie den guten Ruf der einzelnen und der Gruppen sowie die Werte der Kultur verteidigen und schließlich Voraussetzungen dafür schaffen, daß die Ausübung der Religionsfreiheit auch in den Medien gewährleistet ist" (CeP 87).

„Es wird sehr empfohlen, daß die Kommunikatoren sowie andere dem Kommunikationsbereich zugeordnete Institutionen von sich aus Gremien mit eigenen Satzungen schaffen; diese sollen in allen hier auftauchenden Sachfragen beratend tätig werden. In diese Gremien sollen Vertreter aller Gruppen der Gesellschaft berufen werden. So kann man hoffen, daß staatliche Eingriffe und ein Übergewicht wirtschaftlicher Interessen vermieden werden. Andererseits könnte dadurch die Zusammenarbeit unter den Kommunikatoren so verbessert werden, daB die Gesamtanstrengung der Medien für das Gemeinwohl wirksamer wird. Allerdings wird es hier und da wohl nötig sein, daß die öffentliche Hand selbst die Errichtung solcher Kontrollgremien in die Wege leitet, bei denen die repräsentative Vertretung der verschiedenen gesellschaftlichen Gruppierungen gesetzlich geordnet ist“ (CeP 88).

„Communio et Progressio“ erkennt, daB Kommunikationsfreiheit durch bloße Gewährung noch nicht schon gesellschaftlich wirklich wird. Sie muß durch gesetzliche Regelung geschützt werden, damit nicht gesellschaftliche Ungleichheit faktisch zur Beschränkung der Kommunikationsrechte für die schwächeren Gruppen der Gesellschaft führt. Medienpolitik und Kommunikationsgesetzgebung müssen dafür sorgen, daß die Vielfalt der Meinungen in der Massenkommunikation vertreten ist und sich keine gesellschaftliche Gruppe vom „Gespräch am runden Tisch" ausgeschlossen findet. ${ }^{3}$ Die Pastoralinstruktion sieht deutlich die Gefahren, die aus marktwirtschaftlichen GesetzmäBigkeiten für die Widerspiegelung gesellschaftlicher Vielfalt drohen. Sie befürwortet im Bereich der Massenkommunikation die freiwillige oder gesetzlich vorgeschriebene Einrichtung von beratenden und kontrollierenden Gremien, in denen alle gesellschaftlichen Gruppen vertreten sind. So sollen staatliche Einflußnahme und wirtschaftliche Dominanz verhindert werden. Dieser Empfehlung kommt bei uns der öffentlich-rechtliche Rundfunk am nächsten, der in den Rundfunkräten Institutionen gesellschaftlicher Kontrolle mit erheblichen Befugnissen besitzt.

Diese strukturbezogenen ethischen Überlegungen bezieht die Pastoralinstruktion auch auf die internationale Kommunikation bei der genauso das Kriterium der gleichberechtigten Beteiligung aller gilt. 
„Die Träger der öffentlichen Gewalt müssen ihre Aufgaben im Kommunikationsbereich schon heute weltweit sehen. Durch internationale Vereinbarungen muß garantiert werden, daß überall, ohne Rassendiskriminierung und angemaßte Monopolstellungen, ein ausreichendes Kommunikationswesen aufgebaut und entwickelt wird. Auch die Probleme der Satellitenkommunikation müssen durch internationale Verträge geregelt werden. Dann können alle Völker den ihnen gebührenden Platz im weltweiten Gespräch der Menschheit einnehmen“ (CeP 91).

\subsection{Anforderungen an die einzelnen: Handlungsethik}

Natürlich beschränkt sich „Communio et Progressio“ nicht auf die ethisch geforderten strukturellen Bedingungen der Massenkommunikation, sondern entwickelt eine Ethik für die in Massenkommunikation Handelnden: Für Kommunikatoren und Rezipienten.

„Wenn die Instrumente der sozialen Kommunikation den Menschen wirklich dienen sollen, muß vor allem gesehen werden, welchen Anteil der Mensch an ihrem Funktionieren hat; denn seine Rolle ist dabei entscheidender als jede noch so faszinierende mechanische oder elektronische Perfektion. Die Aufgaben, welche die Kommunikationsmittel in der Gesellschaft haben, erfüllen sich nicht von selbst. Deshalb müssen Kommunikatoren und Rezipienten auf diesem Gebiet gut vorbereitet und ausgebildet sein, damit sie die Möglichkeit der Medien voll ausschöpfen können. Jeder muß sich seiner besonderen Rolle bewußt sein und sich darauf als einzelner und als Glied der Gesellschaft vorbereiten. Dem Staat, der Kirche und den Erziehern fallen dabei Verpflichtungen besonderer Art zu, damit die Medien zum Wohl der Gesellschaft tatsächlich leisten, was sie versprechen“" (CeP 63).

Die ethischen Anforderungen an das Handeln der Kommunikatoren werden dabei von der Leistung her bestimmt, die Massenmedien für die Gesellschaft erbringen: Ein Forum für die gesellschaftliche Kommunikation zu sein.

"Die Kommunikatoren sind Anwalt und Stimulator im Gesprăch der Gesellschaft. Sie sind gleichsam Gesprächsleiter am großen runden Tisch, den die Instrumente der sozialen Kommunikation bilden. Es ist daher ihre Aufgabe, immer und überall um die Verwirklichung der Ziele der gesellschaftlichen Kommunikation bemüht zu sein, $d . h$. in jeder Hinsicht den menschlichen Fortschritt zu fördern sowie die Menschen 
zueinander zu führen und sie in wirklicher Gemeinschaft zu verbinden" (CeP 73).

„Bei der Auswahl ihrer Stoffe werden die Kommunikatoren dafür sorgen, alles für die Öffentlichkeit Notwendige zu berücksichtigen. Sie werden genau darauf achten, daß alle gesellschaftlich relevanten Gruppen mit ihren Auffassungen zu Wort kommen. In diesem Zusammenhang sollten sich die Kommunikatoren künftig noch eingehender damit beschäftigen, welches Publikum sie im Einzelfall wohl haben werden und mit ihm auch direkten Kontakt suchen. Nur wenn sie sich einstellen auf die verschiedenen Altersstufen, auf die einzelnen Schichten in der Gesellschaft und den unterschiedlichen Bildungsstand ihrer Rezipienten, können sie allen Erfordernissen und Erwartungen des Publikums gerecht werden. Denn nur zwischen wissenden, freien und verantwortungsbewußten Menschen gibt es jenen ständigen Dialog, den die Instrumente der sozialen Kommunikation ermöglichen" (CeP 74).

„Communio et Progressio“ geht dann noch spezieller auf einzelne Berufsgruppen innerhalb der Kommunikatoren ein. Aber auch den Lesern, Zuhörern oder Zuschauern kommt für das Gelingen der Massenkommunikation eine große Bedeutung zu:

„Die Einflußmöglichkeiten der Rezipienten und damit auch ihre Pflichten sind viel größer, als gemeinhin angenommen wird. Von den Rezipienten hängt es entscheidend $a b$, ob ein wirkliches Gespräch zustande kommt. Bleiben sie passiv und stumm, wird Kommunikation zur Einbahnstraße, auch wenn sich die Kommunikatoren noch so sehr um einen Dialog bemühen“ (CeP 81).

\subsection{Medienpädagogik als Hilfe für sachgerechtes und damit auch ethisch qualifiziertes Handeln}

Ein besonderes Markenzeichen von „Communio et Progressio“ ist die ausführliche Berücksichtigung der Medienpädagogik. Die Pastoralinstruktion erkennt, daß moralische Appelle allein nicht genügen. Den Rezipenten und Kommunikatoren muB Hilfestellung für den sachgerechten Umgang mit den Massenmedien gegeben werden. Angezielt wird keine Bewahrpädagogik - „eine Anleitung zum ,rechten“ Gebrauch des publizistischen Angebots, die in erster Linie beibringen sollte, wovor sich der gut katholische Leser, Hörer, Seher zu hüten 
habe“4 - , sondern ein Gesamtverständnis der Massenkommunikation und ein sachgerechter Umgang mit Medien. Auch hier bleibt der Blick also offen für strukturelle Bedingungen und die gesamtgesellschaftliche Dimension.

„Medienpädagogik ist eine vordringliche Aufgabe. Sie muß die Funktionsprinzipien der Instrumente der sozialen Kommunikation gründlich vermitteln. Was in dieser Instruktion an Grundsätzlichem angesprochen wird, sollte heute Gemeingut aller Menschen sein. Die Kommunikationsmittel leisten erst dann ihren vollen Beitrag zur Entfaltung des Menschen, wenn er deren Wesen und den Umgang mit innen begriffen hat. Wer ihre Bedeutung nur oberflächlich kennt, schmälert leicht den Verfügungsraum seiner Freiheit. Gegenstand dieser Bildungsarbeit muß sein die die Darstellung der besonderen Eigenart der einzelnen Medien, ihrer Organisationsform und Struktur in dem betreffenden Gebiet und schließlich die Darlegung der Grundsätze für den sachgerechten Umgang mit ihnen. All das muß immer im Blick auf den Menschen und die Gesellschaft behandelt werden" (CeP 64).

„Communio et Progressio“ geht dann ausführlich auf die notwendige Medienpädagogik für Rezipienten und die Aus- und Weiterbildung von Kommunikatoren ein.

\section{Die Rolle der Kommunikationsmittel im Leben der Kirche}

Der letzte Teil, der vom Umfang her knapp die Hälfte des Textes ausmacht, beschäftigt sich mit der Rolle der Kommunikationsmittel im Leben der Katholiken und der Kirche.

„In dreifacher Hinsicht sind also die Instrumente der sozialen Kommunikation für die Katholiken bedeutsam: sie helfen der Kirche, sich der heutigen Welt verständlich zu machen; sie fördern das innerkirchliche Gespräch; schließlich vermitteln sie der Kirche das Verständnis für die Mentalität und die Menschen unserer Zeit, denen sie auf Gottes Geheiß die Botschaft vom Heil bringen soll. Dabei wird sie eine Sprache sprechen, die von den Menschen verstanden wird; und sie wird ansetzen bei den Fragen, welche die Menschheit im Innersten bewegen“ (CeP 125). 


\subsection{Der innerkirchliche Dialog}

"Communio et Progressio“ betont die Bedeutung des innerkirchlichen Gesprächs und der daraus entstehenden öffentlichen Meinung in der Kirche. Dort wo der offene Dialog nicht gefördert und zugelassen wird, liegt eine Schuld vor. Für die innerkirchliche Kommunikation gewährt die Pastoralinstruktion eine Freiheit, wie sie Katholiken lange nicht mehr erfahren haben (und heute wohl faktisch immer noch nicht besitzen) und verpflichtet die kirchlichen Obrigkeiten, die Voraussetzungen dafür zu schaffen. Die Pastoralinstruktion fordert deshalb die Sicherung von Meinungs- und Informationsfreiheit innerhalb der Kirche, die Schaffung von Strukturen auf allen Ebenen, die dem Austausch von Meinungen und Informationen dienen (hierzu gehören die verschiedenen Gremien und Massenmedien) und einen ungehinderten Prozeß $B$ öffentlicher Meinungsbildung. Auch für die innerkirchliche Kommunikation (die wir uns eher im hierarchischen Bild der Pyramide vorzustellen geneigt sind) müssen wir uns das Bild vom runden Tisch vor Augen führen. Alle kirchlichen Gruppen, Einrichtungen und Positionen sollen in einen freien Austausch miteinander kommen können.

Was bedeutet die Vorstellung vom runden Tisch beispielsweise für die kirchlichen Bistumszeitungen? Als zentrales Massenmedium eines Bistums haben sie die Vielfalt der katholischen Kirche und die verschiedenen Weisen der Verwirklichung des Glaubens unter den Bedingungen moderner Gesellschaft und angesichts aktueller Herausforderungen widerzuspiegeln und in Austausch zu bringen. Nicht vorstellbar ist es, daß sich in einer Bistumszeitung nur das findet, was den Konzepten der Diözesanleitung entspricht, auch wenn sie deren Träger und Herausgeber ist - von streng lehramtlichen Fragen einmal abgesehen. Sonst nämlich würde jenes Gespräch gerade nicht zustandekommen, das „Communio et Progressio“ fordert. Es wäre zu überlegen, wie jene von der Pastoralinstruktion (vgl. CeP 88) gewünschten Beratungsund Kontrollgremien, in denen die Vielfalt katholischer Gruppierungen repräsentiert ist, für kirchliche Massenmedien aussehen könnten.

„Darum müssen Katholiken sich völlig dessen bewußt sein, daß sie wirklich die Freiheit der Meinungsäußerung besitzen. Diese Freiheit gründet im Glaubenssinn und in der Liebe; im Glaubenssinn, der vom Geist der Wahrheit geweckt und genährt wird, so daß das Gottesvolk unter der Leitung des heiligen Lehramtes in dessen treuer Gefolgschaft den einmal den Heiligen übergebenen Glauben unverlierbar festhält, in inn mit rechtem Urteil immer tiefer eindringt und inn im Leben umfassender zur Auswirkung kommen läBt; in der Liebe aber, weil durch sie die Kommunikationsfreiheit bis zur Freiheit in Christus 
hinausgehoben wird. Christus nämlich befreite uns von den Fesseln der Sünde. Er machte uns damit erst fähig, im Einklang mit seinem Willen frei zu urteilen. Die verantwortlichen kirchlichen Obrigkeiten werden dafür sorgen, daB sich innerhalb der Kirche auf der Basis der Meinungs- und Redefreiheit der Austausch legitimer Ansichten lebendig entfaltet. Darum werden sie Normen und Bedingungen schaffen, die diesem Ziel dienen“ (CeP 116).

„Wenn die Kirche lebendig sein und ihre Aufgaben wirklich erfüllen will, muß es zwischen kirchlichen Autoritäten auf jeder Ebene, katholischen Einrichtungen und allen Gläubigen einen ständigen, wechselseitigen und weltweiten FluB von Informationen und Meinungen geben. Dafür sind entsprechende Institutionen zu schaffen und hinreichend auszustatten z. B. Nachrichtendienst, Pressestellen, Begegnungszentren und Pastoralräte" (CeP 120).

Die Pastoralinstruktion tritt entschieden für die Freiheit des Gesprächs und der öffentlichen Meinungsbildung in der Kirche ein, die allerdings an die Glaubenswahrheiten gebunden ist. Aber das Verständnis der Wahrheit unseres Glaubens und die Verkündigung des Evangeliums in Wort und Tat unter sich ständig wandelnden gesellschaftlichen Bedingungen bleiben verwiesen auf die innerkirchliche Diskussion (vgl. CeP 117). Ohne hier die dogmatische Diskussion über Lehre sowie das Verhältnis von Einheit und Dissens führen zu können, läBt sich entsprechend der Grundintention von „Communio et Progressio“ davon ausgehen: bei der Sicherung der authentischen Interpretation des Glaubens und der Verteidigung gegen Irrtümer in Wort und Tat darf mehr von Eröffnung von Kommunikation erhofft werden darf als von ihrer Beschränkung.

Das Ziel einer kommunikativen und offenen Kirche wird auch in der strikten Begrenzung von Geheimhaltung zum Ausdruck gebracht. Kirchliches Handeln, das der Frohbotschaft dient und von ihr durchdrungen ist, ist so beschaffen, daB es das Licht der Öffentlichkeit nicht zu scheuen braucht. Andernfalls schadet die Kirche ihrem Auftrag. Gerade weil sich die katholische Kirche gesellschaftlich als eine in ihren Entscheidungswegen und Entscheidungen undurchschaubarsten Institutionen darstellt und häufig meint, auf eine Begründung ihrer Beschlüsse verzichten zu können oder zu müssen, ist diese Forderung von höchster Tragweite.

„Wenn bei der Behandlung irgendwelcher Fragen in der Kirche Geheimhaltung geboten ist, gelten die gleichen Grundsätze wie im staat- 
lichen Bereich. Die geistigen Werte, die in der Kirche zum Ausdruck kommen, erfordern es allerdings, daß die Informationen über ihre Absichten und über die Fülle ihrer Tätigkeit mit einem Höchstmaß an Vollständigkeit, Wahrhaftigkeit und Offenheit gegeben werden. Wenn kirchliche Stellen Nachrichten zurückhalten oder nicht in der Lage sind zu informieren, öffnen sie schädlichen Gerüchten Tür und Tor, anstatt die Wahrheit ans Licht zu fördern. Geheimhaltung muB daher unbedingt auf solche Fälle begrenzt bleiben, bei denen es um den Ruf und das Ansehen eines Menschen geht oder andere Rechte einzelner bzw. von Gruppen verletzt würden“ (CeP 121).

\subsection{Dialog zwischen Kirche und Welt5}

Die Kirche ist durch ihren Grundauftrag, das Evangelium in Wort und Tat zu verkünden und Mensch und Welt im Sinne des Evangeliums umzugestalten, geradezu darauf angelegt, ihre Lehre und ihr Handeln offenkundig zu machen, öffentlich zu machen. Sie muB umgekehrt entsprechend begierig darauf sein, die sich ständig verändernde Welt, der sie das Evangelium verkündet, zu verstehen, ihre Chancen und Gefährdungen zu erfassen, die Zeichen der Zeit zu deuten und zu fragen, was sie im Anspruch des Evangeliums bedeuten. Deswegen tritt die Kirche in Dialog mit der Gesellschaft ein, wie es das II. Vatikanische Konzil vorgemacht hat: „Als Zeuge und Künder des Glaubens des gesamten in Christus geeinten Volkes Gottes kann daher das Konzil dessen Verbundenheit, Achtung und Liebe gegenüber der ganzen Menschheitsfamilie, der dieses ja selbst angefügt ist, nicht beredter bekunden als dadurch, daß es mit ihr in einen Dialog eintritt über all diese verschiedenen Probleme." (Gaudium et spes 3)

„Das Gespräch der Kirche beschränkt sich nicht auf die Gläubigen, sondern bezieht die ganze Welt ein. Die Kirche muB ihre Lehre und ihr Wirken offenkundig machen: die Menschen, an deren Schicksal sie ja teilhat, haben ein Recht darauf, und sie selbst ist dazu durch ein klares göttliches Gebot verpflichtet. Ferner ist sie nach dem Wort des II. Vatikanischen Konzils gehalten, die „Zeichen der Zeit zu deuten“; denn auch durch diese spricht Gott, und sie sind ein Dokument der Vorsehung, die darin die Heilsgeschichte weiterhin offenbar macht. Die Kirche muB also wissen, wie alle Zeitgenossen, nicht nur die Katholiken, auf die jeweils neuesten Ereignisse und geistigen Strömungen antworten. Solches Wissen der Kirche wird um so gründlicher sein, je deutlicher die Instrumente der sozialen Kommunikation diese Antworten darstellen" (CeP 122). 
Da dieser Dialog mit der Welt auch auf Massenmedien angewiesen ist, muß die Kirche den Erfordernissen, GesetzmäBigkeiten und Strukturen der Massenkommunikation gerecht werden.

Wenn sie in der Öffentlichkeit präsent sein will, muß sie sich öffnen. Sie muß bereit sein, den Massenmedien vollständige, wahre und genaue Informationen anzubieten, damit diese ihre Aufgabe erfüllen können. Ihre Sprache und Darstellungsweise muß in der modernen gesellschaftlichen Kommunikation verständlich sein.

Voraussetzung dafür ist, daß die Kirche und die Christen die Aufgaben und Gesetzmäßigkeiten massenmedialen Handelns verstehen und daB es Mitarbeiter gibt, die für die Medienarbeit speziell ausgebildet sind. Deswegen kommt auch der Medienpädagogik in der Kirche ein großer Stellenwert zu. „Communio et Progressio“ fordert deshalb ein breites kirchliches Angebot für alle Katholiken, speziell auch für Priester und Ordensleute, für die Repräsentanten der Kirche, für die in der kirchlichen Medienarbeit Tätigen und für den publizistischen Nachwuchs; sie soll stattfinden in Schulen, Hochschulen, Erwachsenenbildung und im Rahmen der theologischen Ausbildung. Auch die Kommunikationsforschung soll an katholischen Hochschulen gefördert werden.

„Die Kirche sieht die dringliche Notwendigkeit, daß sie selbst den Rezipienten eine Medienpädagogik anbietet, die von christlichem Geist getragen ist. Damit dient sie der sozialen Kommunikation selbst; denn nur gut ausgebildete Rezipienten können wirksam mit den Kommunikatoren ins Gespräch kommen und ihre Ansprüche an die Qualität des Kommunikationsangebotes begründet anmelden. Katholische Schulen und Bildungseinrichtungen müssen ihren schwerwiegenden Auftrag auf diesem Gebiet noch viel intensiver erfüllen. Sie sollen die Rezipienten so bilden, daß sie nicht allein gute Leser, Hörer und Zuschauer sind, sondern daß sie selbst sich auch jener „universalen Sprache“ zu bedienen vermögen, die den Medien eigen ist. So werden sie im Vollsinn Bürger des Zeitalters der sozialen Kommunikation, das in der Gegenwart zu beginnen scheint" (CeP 107).

„Künftige Priester und Ordensleute, auch Ordensschwestern, müssen während ihrer Ausbildung mit der Rolle und Auswirkung dieser Medien in der menschlichen Gesellschaft vertraut gemacht und in den Gebrauch dieser Technik eingewiesen werden; sonst blieben sie völlig lebensfern und für ihr späteres Apostolat ungeeignet. Die Vermittlung solcher Kenntnisse muB integraler Bestandteil ihrer Ausbildung sein. Ohne diese Voraussetzung kann überhaupt niemand mehr wirksam apostolisch tätig werden in einer Welt, die täglich stärker von diesen 
Medien geprägt wird. Weiterhin sollen Priester und Ordensleute die Prozesse der Meinungsbildung genau kennen und sich den Gegebenheiten unserer Zeit anpassen. Denn das Wort Gottes ist den Menschen von heute zu verkünden, und schließlich leisten die Medien selbst einen wirksamen Dienst für die Verkündigung. Wer aus dem Nachwuchs für Priester- und Ordensberufe besondere Neigung und Befähigung zur Arbeit in den Kommunikationsmitteln zeigt, soll dazu weiter ausgebildet werden“ (CeP 111).

„Communio et Progressio“ läßt die Frage offen, ob sich die Kirche für den Zugang zur Massenkommunikation auf eigene Medien stützen soll. Die Antwort kann je nach gesellschaftlicher Situation und Medium unterschiedlich ausfallen. Unabhängig davon ist die Beteiligung an der Massenkommunikation mit einem beträchtlichen finanziellen Einsatz verbunden, den die Pastoralinstruktion trotzdem fordert und für den sie die Unterstützung der Gläubigen erwartet.

\section{Würdigung und Ausblick}

Die Pastoralinstruktion „Communio et Progressio“ verstehen wir unzureichend, wenn wir sie als Anleitung zum Umgang der Kirche mit modernen Massenmedien interpretieren. Denn wo sie sich mit den Kommunikationsmitteln im Leben der Katholiken befaBt, ist die Kirche in ihrer kommunikativen Gestalt das Thema. Sie bietet so eine Lehre über die Kirche von der Kommunikation her. Sie kann sich dabei auf das Kirchenverständnis des II. Vatikanischen Konzils („Lumen gentium“ nach innen, „Gaudium et spes“ nach außen) stützen und denkt es in kommunikativer Hinsicht weiter. „Communio et Progressio“ enthält eine „Kommunikationsverfassung der Kirche“.

Ähnlich intendiert die Pastoralinstruktion in ihrem zweiten Teil Grundsätze für die gesellschaftliche Kommunikation. Sie geht von der Voraussetzung einer pluralistischen Gesellschaft aus und fordert einen wirklichen Pluralismus, der die Gleichberechtigung aller gesellschaftlichen Gruppen realisiert. In gesellschaftlicher Kommunikation geschieht der Zusammenhalt der pluralistischen Gesellschaft, kann sich Solidarität entwickeln und ausdrücken und kommt die Anerkennung der Gleichberechtigung und Unverfügbarkeit der anderen zum Ausdruck. Das christliche Zentralgebot: „Du sollst Deinen Nächsten lieben wie Dich selbst" erfährt in einer Öffentlichkeit, die diesen Namen im Vollsinn verdient, eine gesellschaftliche Einlösung. Die Verkündigung des Evangeliums in Wort und Tat geschieht nicht nur in Kommunikation, sondern bedeutet auch Veränderung von Kom- 
munikation. Weil die Evangelisierung bei der Selbstevangelisierung der Kirche beginnt, wie es Paul VI. in „Evangelii nuntiandi“ dargelegt hat, bedeutet Evangelisierung die Umgestaltung von Kommunikation in Gesellschaft und Kirche.

Die Pastoralinstruktion „Communio et Progressio“ ist durch die gesellschaftliche, kirchliche oder publizistische Entwicklung der letzten 20 Jahre nicht überholt; sie ist nicht eingeholt. Sie bedarf nicht der Ablösung, sondern der Einlösung. Mögen einzelne Artikel in ihren Formulierungen und Detaillösungen diskussionswürdig und revisionsbedürftig sein, wegweisend bleibt die Grundeinsicht und -konzeption.

Die Umsetzung in die kirchliche Wirklichkeit und katholisches Denken scheint eher den umgekehrten Weg gegangen zu sein. Konkrete Regelungen wurden übernommen: Der Aufbau der geforderten Organisationseinheiten, die Einrichtung neuer Stellen und die Durchführung des „Mediensonntags“ ist geschehen. Ebenso hat die Kirche auch gelernt, die Massenmedien in den Dienst zu nehmen und besitzt umfassende publizistische Möglichkeiten. Jedoch scheint gerade das Herzstück von „Communio et Progressio“, nämlich das Verständnis der Aufgabe von Kommunikation und Massenkommunikation, das katholische Denken nicht dauerhaft geprägt zu haben 6 - sofern die Pastoralinstruktion überhaupt gründlich zur Kenntnis genommen und rezipiert wurde.

Zwei Haltungen gewinnen gegen die Zielsetzung des II. Vatikanischen Konzils und der Pastoralinstruktion wieder an Gewicht:

1. Im Gegensatz zur kommunikativen Offenheit und dialogischen Struktur, die das II. Vatikanische Konzil und „Communio et Progressio“ auszeichnen, scheinen sich Ängste vor zu großer Kommunikations-und Medienfreiheit auszubreiten, die dazu führen können, kirchliche Kommunikation obrigkeitlich zu steuern und zu begrenzen. ${ }^{7}$ Dies wäre ein Rückfall in den vormodernen und vorkonziliaren Versuch, Wahrheit gegen Freiheit ,auszuspielen“ und Wahrheit mit "Macht" durchzusetzen. Eine auf Macht beruhende strategische Steuerung von Kommunikation und Information ist in Politik und Wirtschaft weithin üblich. Aber sie entspricht nicht der Kommunikationsverfassung, die "Communio et Progressio“ uns vorstellt: nicht für die Gesellschaft und schon gar nicht für die Kirche.

2. Ebenso steht das damit eng verbundene instrumentelle Verständnis der Massenmedien nicht in Einklang mit „Communio et Progressio“ auch wenn sie in der Pastoralinstruktion die etwas unglückliche Bezeichnung „Instrumente der sozialen Kommunikation“ tragen. Massenmedien erscheinen nur noch als Mittel für beliebige Ziele. Die Rechtfertigung des Mediengebrauchs erfolgt vom Zweck her: Der Me- 
diengebrauch ist gut, wenn die Sache, um die es geht, gut ist, oder wenn die Gruppen, die sich der Medien bedienen, gut sind. Im Sinne dieses Verständnisses käme es darauf an, kirchliche Medienpräsenz zu maximieren. Aber entscheidend für die Qualität der gesellschaftlichen Kommunikation ist das „Wie“ der Kommunikation. Davon hängt es nämlich ab, ob überhaupt soziale Kommunikation zustandekommt, die zu „communio et progressio“ beiträgt. Um es noch einmal am Extrem zu verdeutlichen: Während die instrumentalistische Zerrform des Verständnisses von Massenkommunikation möglichst viel öffentliche Präsenz der Kirche im Einsatz für das Evangelium erkämpfen zu müssen meint, belehrt uns ein entwickeltes Verständnis von Massenkommunikation, daß die Umgestaltung gesellschaftlicher Kommunikation im Sinne des Evangeliums von der Kirche das Eintreten für die Kommunikationsmöglichkeiten aller, sogar der Kirchenkritiker, verlangt. ${ }^{8}$

Kirchliche Medienarbeit hat entsprechend drei Aufgaben:

- Sie sorgt dafür, daß Kirche als qualifizierter und kommunikativer Gesprächspartner ihren Beitrag vernehmlich und verständlich in das öffentliche Gespräch der Gesellschaft einbringen kann.

- Sie setzt sich in „kommunikativer Diakonie“9 dafür ein, daß eine öffentliche Kommunikation in der Gesellschaft zustandekommt, die dem Bild vom „runden Tisch“ gerecht wird, an dem alle, auch die politisch und wirtschaftlich Schwächeren, Gehör finden.

- Sie sorgt für einen offenen und freien innerkirchlichen Dialog.

Das Eintreten von „Communio et Progressio“ für eine freie und offene Kommunikation gründet im Wissen, daß die Botschaft des Glaubens Transparenz und Öffentlichkeit nicht zu scheuen hat und in freier Kommunikation und Argumentation bestehen kann. Mehr als jede Kirchenkritik der Kirche schaden kann, kann sie sich selber schaden, wenn sie - aus Angst vor ihr - Kommunikation zu begrenzen und zu kontrollieren sucht. Nicht zu große Offenheit und Öffentlichkeit, sondern zu groBe Geschlossenheit und Verschlossenheit sind in der gegenwärtigen Situation die Gefahren, die den Erfolg der Evangelisierung in Frage stellen können. 


\section{Anmerkungen:}

1 Dies war schon wăhrend der Beratungen von „Inter mirifica“ erkennbar. Aber die harte Geschäftsordnung des Konzils erlaubte es nicht, einen einmal im Grundsatz akzeptierten Entwurf nochmals substantiell zu verändern. So zeigen die Abstimmungen eine wachsende Unzufriedenheit der Konzilsvăter mit diesem Text, ohne daB diese zur Konsequenz eines neuen Entwurfs führen konnte. (Vgl. die Einleitung zum Dekret über die sozialen Kommunikationsmittel „Inter mirifica“. In: Karl Rahner/Her. bert Vorgrimler: Kleines Konzilskompendium. Freiburg ${ }^{14} 1980$, S. 91 - 94.

2 Georg Moser: Kommunikation und Menschlichkeit. In: Communicatio Socialis 16 (1983) 191 - 199, 194. Vgl. auch: Kommunikation und Menschlichkeit. Aus Veröffentlichungen von Bischof Georg Moser. In: Heinz Glăssgen/Hella Tompert (Hg): Zeitgespräch. Kirche und Medien. Freiburg 1988, S. 184 - 188.

3 Zu den strukturellen Bedingungen der Massenkommunikation vgl. ausfuhrlicher Bernhard Laux: Die institutionelle Ordnung der Massenkommunikation aus sozialethischer Sicht. Frankfurt 1986

4 Michael Schmolke: Zehn ideengeschichtliche Beobachtungen zur Pastoralinstruktion „Communio et Progressio“, in: Franz-Josef Eilers u. a. (Hg.): Kirche und Publizistik. 13 Kommentare zur Pastoralinstruktion „Communio et Progressio“ mit dem deutschen Originaltext. Paderborn 1972, S. 17 - 25, 22.

5 Man darf über den abkürzenden Formulierungen wie „Kirche und Welt" oder „Kirche und Gesellschaft" nicht vergessen, daß Kirche „Kirche in der Welt" und in der Gesellschaft ist und ihnen nicht von außen entgegentritt.

6 So auch Michael Schmolke: Braucht die Kirche eine neue Medienpolitik? In: Communicatio Socialis 23 (1990) H. 3, S. 197 - 206. Michael Schmolke hat die Pastoralinstruktion bei ihrem Erscheinen und an ihrem zehnten „Geburtstag“ kommentiert.

7 So ist im Öffentlichkeitsverständnis der lehramtlichen „Instruktion über die kirchliche Berufung der Theologen" von Geist und Buchstaben des lehramtlichen Textes „Communio et Progressio" nichts zu spüren. Vgl. Kongregation für die Glaubenslehre: Instruktion über die kirchliche Berufung der Theologen (24. 5. 1990), hg. v. Sekretariat der Deutschen Bischofskonferenz Bonn (Verlautbarungen des apostolischen Stuhls 98); zu ihrem Öffentlichkeitsverständnis vgl. Helmuth Rolfes: Lehramt, Theologie und Öffentlichkeit. Überlegungen zum Öffentlichkeitsverständnis der „Instruktion über die kirchliche Berufung des Theologen ". In: Communication Socialis $23(1990) \mathrm{H}$. 3, S. $207-216$.

$8 \mathrm{Vgl}$. auch die Erklärung des II. Vatikanischen Konzils über die Religionsfreiheit „Dignitatis humanae“, Nr. 2, 6 und 13.

9 Peter Düsterfeld: Kommunikative Diakonie. Überlegungen zum Verhältnis der Kirche zu den Massenmedien. In: Funk-Korrespondenz Nr. 13-14/31. 3. 1988, S. 1 - 6. 